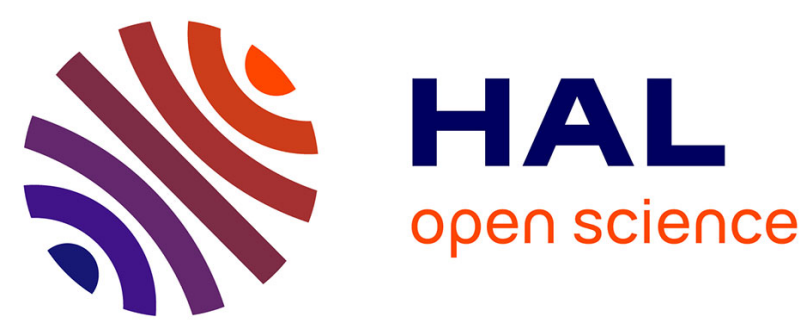

\title{
Long-term outcome of ovarian function in women with intermittent premature ovarian insufficiency
}

Anne Bachelot, Carole Nicolas, Maud Bidet, Jérôme Dulon, Monique Leban, Jean Louis Golmard, Michel Polak, Philippe Touraine

\section{To cite this version:}

Anne Bachelot, Carole Nicolas, Maud Bidet, Jérôme Dulon, Monique Leban, et al.. Long-term outcome of ovarian function in women with intermittent premature ovarian insufficiency. Clinical Endocrinology, 2016, 10.1111/cen.13105 . hal-01327895

\section{HAL Id: hal-01327895 \\ https://hal.sorbonne-universite.fr/hal-01327895}

Submitted on 7 Jun 2016

HAL is a multi-disciplinary open access archive for the deposit and dissemination of scientific research documents, whether they are published or not. The documents may come from teaching and research institutions in France or abroad, or from public or private research centers.
L'archive ouverte pluridisciplinaire HAL, est destinée au dépôt et à la diffusion de documents scientifiques de niveau recherche, publiés ou non, émanant des établissements d'enseignement et de recherche français ou étrangers, des laboratoires publics ou privés. 


\section{Long-term outcome of ovarian function in women with intermittent premature ovarian insufficiency}

Short Title: Follow up of ovarian function of POI patients

Keywords: premature ovarian insufficiency; ovarian function; pregnancy; fertility

Anne Bachelot ${ }^{* \dagger}$ a , Carole Nicolas ${ }^{* \dagger}$ a , Maud Bidet ${ }^{\ddagger}$, Jérôme Dulon*, Monique Leban", Jean Louis Golmard $^{\dagger \S}$, Michel Polak ${ }^{\ddagger \pm}$, Philippe Touraine ${ }^{* \dagger}$

*AP-HP, IE3M, Hôpital Pitié-Salpêtrière, Department of Endocrinology and Reproductive Medicine and Centre de Référence des Maladies Endocriniennes Rares de la croissance et Centre des Pathologies gynécologiques Rares, ICAN, Paris, France

${ }^{\dagger}$ UPMC Univ Paris 06, Paris, France

‡ AP-HP, Department of Pediatric Endocrinology, Gynecology and Diabetology, Centre de Référence des Maladies Endocriniennes Rares de la Croissance et Centre des pathologies gynécologiques Rares, Hôpital Universitaire Necker Enfants malades, Paris, France "AP-HP, Hôpital Pitié-Salpêtrière, Department of Hormonal Biochemistry, Paris, France

§ AP-HP, Hôpital Pitié-Salpêtrière, Clinical Research Unit, Paris, France

${ }^{ \pm}$Université Paris Descartes, Paris 05, Paris, France

${ }^{a}$ A.B. and C.N. contributed equally to this work. 
Correspondence: Philippe Touraine, MD, PhD, IE3M, Department of Endocrinology and Reproductive Medicine, Groupe Hospitalier Pitié-Salpêtrière, 47-83, boulevard de l’Hôpital, 75013 Paris, France.

Phone: +33142160254

Fax: +33142160255

E-mail: philippe.touraine@aphp.fr

Declaration of Interest: nothing to declare

Word Count: Abstract (247) and Main Text (2643) 


\section{SUMMARY}

Context: Spontaneous resumption of ovarian function is not a rare phenomenon in patients with premature ovarian insufficiency (POI). The outcome of this resumption is not known.

Objective: To describe the outcome following the resumption of ovarian function in POI patients.

Design: Cross sectional study

Setting: University medical centre

Patients and main outcome measures: Cumulative incidence of ovarian function resumption and risk factors arresting this resumption during follow-up were determined in a large cohort of POI women.

Results: Five hundred and seven patients were included in the study, with a follow up of $3.44 \pm 4.05$ years [0-29]. Of these, 117 (23\%) had features of ovarian function resumption. The cumulative incidence of pregnancy was 3.5\% among the whole cohort and $15.3 \%$ among patients with resumption of ovarian function. Fifty-five patients (47\%) experienced an arrest of their resumption during the follow up period. In univariate analysis, high FSH and DHEA levels at initial evaluation were risk factors for the arrest of the resumption of ovarian function. In multivariate analysis, high FSH levels at the initial evaluation (1.89 [1.10-3.23], p=0.03) and older age at diagnosis (1.53 [1.01-2.33], $\mathrm{p}=0.04)$ were risk factors for the arrest of this resumption.

Conclusion: Resumption of ovarian function is not a rare or brief phenomenon in POI women. The identification of predictive factors of this resumption, as well as its duration, increases our knowledge of the natural history of POI, and will improve the medical management, especially infertility counselling of these patients. 


\section{INTRODUCTION}

Premature ovarian insufficiency (POI) is a disorder affecting approximately $1 \%$ of women under 40 years of age. ${ }^{1}$ POI encompasses a heterogeneous spectrum of conditions, through two major mechanisms: follicle dysfunction and follicle depletion. ${ }^{2}$ Although causes such as autoimmunity, monosomy $\mathrm{X}$ and environmental factors play a role in POI, the aetiology in most cases remains unknown. ${ }^{3}$

POI is not premature menopause. Indeed, resumption of ovarian function has been reported in nearly a quarter of affected women. ${ }^{4-8}$ We have previously found, by histological examination of ovarian biopsies in these women, that some (28\%) exhibit the presence of growing follicles. ${ }^{3}$ Moreover, in cross-sectional studies, approximately 3 to $10 \%$ are reported to conceive spontaneously after diagnosis. ${ }^{8}$ We recently demonstrated that spontaneous resumption of ovarian function is not a rare phenomenon in POI patients since it occurs in $25 \%$ of cases 48 months after diagnosis. ${ }^{4}$ Moreover, spontaneous pregnancy occurred in $4.4 \%$ of patients with POI. Our study led to the identification of predictive factors for the resumption: family history, secondary amenorrhoea, presence of follicles at ultrasonography, and inhibin B and estradiol concentrations. ${ }^{4}$ These parameters were independent predictive factors for resumption of ovarian activity in POI patients and using the Cox model we created a predictive score. ${ }^{4}$ However, a remaining question from this study was the outcome of these patients with ovarian function resumption, which is actually not known.

We therefore designed a follow-up study of POI women after their first evaluation in a larger cohort, to assess the prevalence and long term outcome of ovarian function resumption and to identify risk factors for the arrest of this resumption. 


\section{PATIENTS AND METHODS}

\section{Patients}

A cohort of POI patients was set up in our department in 2001. This included idiopathic POI patients referred to our department since 1997, and included 542 consecutive patients in December 2013. These patients were studied retrospectively before 2001 and thereafter prospectively. Idiopathic POI was defined by at least 4 months of amenorrhoea in women under 40 years, with two FSH levels above 30 IU/L at an interval of at least 1 month. Patients with Turner’s syndrome, 46 XY gonadal dysgenesis or women with a history of secondary POI (such as chemotherapy, pelvic radiotherapy or ovarian surgery) were excluded. Every woman with POI diagnosis was prescribed with hormonal substitution therapy, but for various reasons, some of them did not or stopped taking it. The Institutional Review Board approved the study and informed consent was obtained from all patients. Three hundred and fifty-six patients in this cohort were previously described, ${ }^{3}$ as were their clinical and hormonal status including determination of FSH, LH, estradiol, inhibin B (from 1997) and anti-Mullerian hormone (AMH) concentrations (from 2003). Pelvic ultrasonography was used to document the presence or absence of follicles, maximum ovarian length and ovary area. Genetic screening included karyotype determination and DNA extraction for FMR1 gene.

Medical files were available for 507 patients of our cohort; they represent our active cohort of POI patients who will be considered later in this study. Medical files were examined in June 2013 to study the natural history of ovarian function in these patients during follow up. Data were collected as follows: in the whole cohort, 181 patients were regularly followed in our department or contacted by mail or by phone between January 2012 and December 2013. For the remaining 326 patients, the same data were collected, but from their clinical files before 2013. The follow 
up period was defined as the duration between the first evaluation in our centre and last news obtained by medical files or contact with the patient by mail or phone. Resumption of ovarian function was defined as in our previous work, by the presence of a spontaneous pregnancy and/or resumption of menstrual cycles (at least 2 consecutive uterine bleeds at intervals of 3 to 6 weeks) without any hormonal treatment and/or a FSH level $<13$ IU/L (upper normal range in our laboratory) after diagnosis, all without any hormonal replacement therapy. ${ }^{4}$ Arrest of the resumption was defined by the disappearance of the signs of resumption or HRT prescription.

\section{Hormone measurements}

Blood was collected and plasma was immediately separated by centrifugation for 15 min at $4 \mathrm{C}$.

Plasma LH and FSH were measured by immunofluorometric assay (Cis-Bio, Gif-sur-Yvette, France). The intra- and interassay coefficients of variation were 1.5 and $5.2 \%$ for $\mathrm{LH}$ and 2.6 and 4\% for FSH, respectively. The detection limit was $0.15 \mathrm{IU} / \mathrm{l}$ for both assays. Estradiol was determined after plasma extraction (DiaSorin, Antony, France; Schering CisBio International, Gif Sur Yvette, France). Plasma inhibin B was measured using a commercial ELISA (Serotec, Kidlington, Oxford, UK) with a detection limit of $10 \mathrm{ng} / \mathrm{l}$ and intra- and interassay coefficients of variation of 6 and 15\%, respectively. Inhibin B level was considered to be $5 \mathrm{ng} / \mathrm{l}$ when the level was below $10 \mathrm{ng} / \mathrm{l}$. Serum AMH concentrations were measured in duplicate using AMH/MIS Elisa ${ }^{\circledR}$ Immunotech, Beckman-Coulter from 2006 to 2009 and EIA AMH /MIS ${ }^{\circledR}$ Immunotech, Beckman-Coulter from 2009 to 2014. Samples collected before 2006 were frozen at -20 C until assay. The intra- and interassay coefficients of variation for AMH determination were 5.3 and 8.7\%, respectively. The AMH assay sensitivity was $0.05 \mu \mathrm{g} / \mathrm{l}$. In women with normal menstrual cycles, the normal range for day $3-5$ of the cycle is $2.2-6.8 \mu \mathrm{g} / \mathrm{l}$. Testosterone concentrations were determined by RIA (Orion Diagnostica, Espoo, Finland). The intra- and interassay coefficients of variation were between 3.8 and $7.5 \%$, and between 4.8 and 7\%, respectively. The 
testosterone assay sensitivity was $0.1 \mathrm{nmol} / \mathrm{l}$ (range of measurement, 0.5 to $50 \mathrm{nmol} / \mathrm{l}$ ).

Androstenedione concentrations were determined by RIA (Immunotech Beckman-Coulter, Marseille, France). The intra- and interassay coefficients of variation were $8.1 \%$ or less and 11.9\% or less, respectively. Plasma dehydroepiandrosterone (DHEA) was measured by conventional RIA (Immunotech Beckman-Coulter, Marseille, France). The intra-assay coefficient ranged from 2.6 to $8.1 \%$, and the interassay coefficient ranged from 3.7 to $11.9 \%$. The normal range was based on results obtained from healthy women during a normal menstrual cycle and provided by our hormonal investigation laboratory.

\section{Ovarian ultrasonography}

Pelvic ultrasound was performed using a Siemens Sonoline Elegra ultrasound system and a 6.5MHz probe. When available, ovarian surface was calculated as follows: $\mathrm{S}=\mathrm{L}$ (length) $\times \mathrm{W}$ (width) $\times 0.8$. The normal ovarian area is between 2 and $6 \mathrm{~cm}^{2}$.

\section{Statistical analysis}

Descriptive statistics used means \pm sd or median [interquartile interval] for quantitative variables and numbers (percentages) for qualitative ones. Resumption of ovarian function (ROF) and duration of resumption (DR) were analysed in the framework of survival analysis, with the delay between diagnosis and ROF considered as the time to event for ROF and the time between the beginning and the arrest of ROF considered as the time to event for DR. The time of the last follow-up was considered as the censoring time for ROF when no ROF occurred. The cumulative incidence of ROF was estimated by one minus the ROF survival function, estimated by the Kaplan-Meïer method. DR survival function was estimated by the Kaplan-Meïer method. Risk factors for the arrest of ROF were assessed using two steps: in a first step a univariate analysis 
was performed by univariate Cox models. In a second step, a stepwise regression was performed with multivariate Cox models. All the variables with a p-value lower than 0.10 in the univariate analysis were entered in the stepwise regression and the variables with a p-value $<0.05$ by the Wald test were retained in the final models. All tests were two-sided, with a p-value $<0.05$ considered as significant. Statistical analysis was performed using the SAS 9.3 software package (SAS Institude Inc, Cary, NC).

\section{RESULTS}

Among the 507 patients initially included in this study, 122 had features of resumption of ovarian function. Medical files documenting their resumption were available for only 117 of these patients (23\% of the cohort) (Fig. 1). The mean age was $30.1 \pm 6.4$ years at POI diagnosis

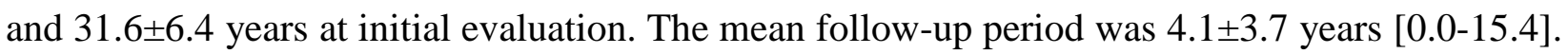
Biochemical data of the patients are presented in Table 1. Most of our patients, $98.2 \%$ ( $n=115$ ) presented with secondary amenorrhea. Of the 117 patients with resumption of ovarian function, 101 (87\%) experienced resumption of menstrual cycles and 60 (51.3\%) a decreased FSH level below the upper limit of the normal range. The cumulative incidence of spontaneous pregnancy $(\mathrm{n}=18)$ was $15.3 \%$ among the patients with resumption of ovarian function, representing $3.5 \%$ of the whole cohort. Nearly 50\% of patients experienced only one sign of resumption, whereas $7.6 \%$ experienced three signs. The cumulative incidence of POI patients with resumption of ovarian function was 23\% at 4 years, 28\% at 10 years and 32\% at 20 years after diagnosis in KaplanMeier analysis (Fig. 2). Fifty nine percent of the patients with resumption experienced this event in the first year after diagnosis (Fig. 2). The cumulative incidence of pregnancy is shown in Fig.3. 


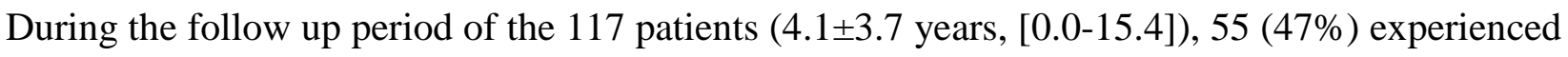
an arrest of resumption of ovarian function. One year after resumption, $75 \%$ of the patients continued to have signs of ovarian function, $44 \%$ after 5 years and 26\% after 10 years (Fig. 4). Risk factors associated with the arrest of resumption of ovarian function in the univariate analysis are shown in Table 2. High FSH and DHEA concentrations at first evaluation (B1) were found to be associated risk factors for the arrest of the resumption.

In multivariable analysis, the final model included the following parameters: age at diagnosis, FSH, LH, DHEA and AMH concentrations at B1. The choice of these parameters entering in the final model was based on the results of univariate analyses and the missing data rates. High FSH levels at the initial evaluation and older age at diagnosis were risk factors for the arrest of this resumption: hazard ratio of FSH leve1s at B1 was 1.89 [1.10-3.23], $\mathrm{p}=0.03$, hazard ratio of age at diagnosis was 1.53 [1.01-2.33], $\mathrm{p}=0.04$.

\section{DISCUSSION}

Observational and interventional studies have reported ovulation and spontaneous pregnancies in POI patients. ${ }^{4-8}$ However, these studies do not have homogeneous inclusion criteria and most of them included a small number of patients. There is, to date, no information in the literature on the duration of ovarian resumption in POI patients or on its predictive factors. In our cohort of 507 POI women, the largest cohort described with extensive clinical, biological, ultrasound and genetic data, we reported the natural history of this resumption and searched for risk factors for this arrest. 
From the cohort, the cumulative incidence of POI patients with resumption of ovarian function was $23 \%$; these results are consistent with the literature. ${ }^{3-17}$ The cumulative incidence of pregnancies in our whole cohort of POI patients was 3.5\% during the follow up period, in accordance of the literature, ${ }^{8-17}$ and representing $15.3 \%$ of our POI patients with resumption of ovarian function. The resumption occurs for most patients during the year following diagnosis.

During the follow up period in women who had resumed function, 47\% experienced an arrest of ovarian function. At one year after resumption, $75 \%$ of the patients continued to have signs of ovarian function. Univariate analysis showed that higher FSH and DHEA levels were risk factors for arrest following the resumption of ovarian function. Persistent ovarian function was thus present in POI women with lower FSH at diagnosis, which represent a subgroup of patients with persistent follicle reserve, with inhibin B, and estrogen exerting control on FSH levels. Inhibin B produced by granulosa cells has been described as a marker for the growth of the small antral follicle cohort (18). FSH levels are dependent on underlying follicular activity and closely related to inhibin B secretion. ${ }^{18}$ It therefore seems logical that low FSH levels in POI patients are associated with some ovarian activity. As the size of the follicle pool declines, inhibin B levels fall and FSH levels rise. The latter reflects loss of inhibin restraint. ${ }^{18}$

Androgen production is decreased in POI women, especially testosterone and androstenedione, but not DHEA-S and DHEA, compared with age-matched fertile controls. ${ }^{19,20}$ In our previous study, we showed that DHEA levels were predictive of the resumption of ovarian function. ${ }^{4}$ In the present study, higher levels of DHEA appear as a risk factor for arrest following the resumption of ovarian activity. However, in multivariate analysis DHEAS lost its significance. Recently, a study on the effects of ovarian tissue exposure to DHEA supplementation in vivo was conducted in female sheep. ${ }^{21}$ The authors showed that a higher 
proportion of the follicle population was observed to be in the antral stage after DHEA exposure. The observation coincided with an increase in the rate of primordial follicle initiation and preantral follicle development. ${ }^{21}$ These data support the idea that DHEA might be useful to delay the effects of ovarian ageing. A possible explanation is that androgens could promote the resumption of ovarian function, but could also be a risk factor for early exhaustion of the remaining follicles in women with POI, explaining the arrest of this resumption. This should be further and more precisely evaluated.

Here, AMH level was not a predictive factor for the arrest of the resumption of ovarian function. In perimenopausal stages, AMH declines well before FSH increases. ${ }^{18,22}$ AMH assays used in this study are perhaps not sensitive enough to discriminate patients with a low ovarian reserve. Indeed, AMH level seems to be more a marker than a predictor of ovarian function, as has recently been reported in patients with Turner syndrome. ${ }^{23}$ In POI patients with a low ovarian reserve and very low or undetectable AMH levels, one can speculate that a low FSH level reflects follicular estradiol and inhibin B production and suggests a better follicle quality. Surprisingly, the absence of follicles on ovarian ultrasonography was not a predictive factor for the arrest of the resumption of ovarian function. This could be explained by the fact that the ultrasonography included in the analysis was performed at POI diagnosis, but not during follow up.

A fairly high proportion of women will experience some evidence of ovarian function after diagnosis of ovarian insufficiency. The resumption of ovarian activity was more frequently observed in the first year after POI diagnosis, as in our previous study. ${ }^{4}$ This justifies the term insufficiency rather than premature menopause. Indeed, there cannot be a presumption that a woman will never experience some recovery of ovarian function. The knowledge of cumulative incidence of resumption and spontaneous pregnancy could be useful for clinicians providing 
advice and counselling for women with POI. In multivariate analysis, the risk factors for the arrest of resumption of ovarian function were high FSH levels in the first evaluation and an older age at POI diagnosis. This phenomenon probably represents POI patients that were diagnosed late, in a period during which very few follicles were still present in the ovaries.

Our study has some bias: POI time of onset, the recovery of ovarian function and its duration were sometimes difficult to establish in our study. Indeed, hormone replacement therapy eclipses the ability to diagnose spontaneous normalization of FSH. Moreover, the most frequent criterion was the resumption of menstrual cycles (87\%), but most of the POI patients were receiving HRT and a resumption of ovarian activity could not be detected in these women. Finally, as part of our study was retrospective, it could potentially underestimate the number of fluctuating cases. These results highlight the importance of an early diagnosis of POI, in order to monitor and support follicular growth and ovulation when ovarian function still exists. Furthermore, patients with incipient POI should be encouraged to conceive as soon as possible if they wish to have family, or failing that, consider egg or embryo cryopreservation.

In conclusion, our study confirms that spontaneous resumption of ovarian function is not a rare or brief phenomenon in POI women. The identification of predictive factors for this resumption, as well as its duration increases our knowledge of the natural history of POI, and will improve the medical management, especially the infertility management, of these patients. 


\section{REFERENCES}

1 Beck-Peccoz, P. \& Persani, L. (2006) Premature ovarian failure. Orphanet Journal of Rare Disease;1, 9.

2 Nelson, L.M. (2009) Clinical practice. Primary ovarian insufficiency. New England Journal of Medicine, 360, 606-614.

3 Bachelot, A., Rouxel, A., Massin, N. et al. (2009) Phenotyping and genetic studies of 357 consecutive patients presenting with premature ovarian failure. European Journal of Endocrinology, 161, 179-187.

4 Bidet, M., Bachelot, A., Bissauge, E. et al. (2011) Resumption of ovarian function and pregnancies in 358 patients with premature ovarian failure. Journal of Clinical Endocrinology and Metabolism, 96, 3864-3872.

5 Rebar, R.W. \& Connolly, H.V. (1990) Clinical features of young women with hypergonadotropic amenorrhea. Fertility and Sterility, 53, 804-810.

6 Nelson, L.M., Anasti, J.N. \& Kimzey, L.M. et al. (1994) Development of luteinized graafian follicles in patients with karyotypically normal spontaneous premature ovarian failure. Journal of Clinical Endocrinology and Metabolism, 79, 1470-75.

7 Conway, G.S., Kaltsas, G., Patel, A. et al. (1996) Characterization of idiopathic premature ovarian failure. Fertility and Sterility, 65, 337-341.

8 van Kasteren, Y.M. \& Schoemaker, J. (1999) Premature ovarian failure: a systematic review on therapeutic interventions to restore ovarian function and achieve pregnancy. Human Reproduction Update, 5, 483-492. 
9 Nelson, L.M., Kimzey, L.M., White, B.J. et al. (1992) Gonadotropin suppression for the treatment of karyotypically normal spontaneous premature ovarian failure: a controlled trial. Fertility and Sterility, 57, 50-55.

10 Taylor, A.E., Adams, J.M., Mulder, J.E. et al. (1996) A randomized, controlled trial of estradiol replacement therapy in women with hypergonadotropic amenorrhea. Journal of Clinical Endocrinology and Metabolism, 81, 3615-3621.

11 Kreiner, D., Droesch, K., Navot, D. et al. (1988) Spontaneous and pharmacologically induced remissions in patients with premature ovarian failure. Obstetrics and Gynecology, 72, 926-928.

12 Bidet, M., Bachelot, A. \& Touraine, P. (2008) Premature ovarian failure: predictability of intermittent ovarian function and response to ovulation induction agents. Current Opinion in Obstetrics and Gynecology, 20, 416-420.

13 Wallace, W.H. \& Kelsey, T.W. (2004) Ovarian reserve and reproductive age may be determined from measurement of ovarian volume by transvaginal sonography. Human Reproduction, 19, 1612-1617.

14 van Kasteren, Y.M., Hoek, A. \& Schoemaker, J. (1995) Ovulation induction in premature ovarian failure: a placebo-controlled randomized trial combining pituitary suppression with gonadotropin stimulation. Fertility and Sterility, 64, 273-278.

15 Surrey, E.S., Cedars, M.I. (1989) The effect of gonadotropin suppression on the induction of ovulation in premature ovarian failure patients. Fertility and Sterility, 52, 36-41.

16 Tartagni, M., Cicinelli, E., De Pergola, G. et al. (2007) Effects of pretreatment with estrogens on ovarian stimulation with gonadotropins in women with premature ovarian failure: a randomized, placebo-controlled trial. Fertility and Sterility, 87, 858-61. 
17 Badawy, A., Goda, H. \& Ragab, A. (2007) Induction of ovulation in idiopathic premature ovarian failure: a randomized double-blind trial. Reproductive Biomedicine Online, 15, 215-219.

18 Sowers, M.R., Eyvazzadeh, A.D., McConnell, D. et al. (2008) Anti-mullerian hormone and inhibin $\mathrm{B}$ in the definition of ovarian aging and the menopause transition. Journal of Clinical Endocrinology and Metabolism, 93, 3478-3483.

19 Benetti-Pinto, C.L., Bedone, A.J. \& Magna, L.A. (2005) Evaluation of serum androgen levels in women with premature ovarian failure. Fertility and Sterility, 83, 508-510.

20 Bachelot, A., Meduri, G., Massin, N. et al. (2005) Ovarian steroidogenesis and serum androgen levels in patients with premature ovarian failure. Journal of Clinical Endocrinology and Metabolism, 90, 2391-2396.

21 Narkwichean, A., Jayaprakasan, K., Maalouf, W.E. et al. (2014) Effects of dehydroepiandrosterone on in vivo ovine follicular development. Human Reproduction, 29, 146-154.

22 Van Disseldorp, J., Faddy, M.J., Themmen, A.P. et al. (2008) Relationship of serum antimullerian hormone concentration to age at menopause. Journal of Clinical Endocrinology and Metabolism, 93, 2129-2134.

23 Lunding, S.A., Aksglaede, L., Anderson, R.A. et al. (2015) AMH as Predictor of Premature Ovarian Insufficiency: A Longitudinal Study of 120 Turner Syndrome Patients. Journal of Clinical Endocrinology and Metabolism, 100, E1030-1038. 


\section{Figure Legends:}

Figure 1: POI patients included in the study

Figure 2: Estimated cumulative incidence of POI patients with resumption of ovarian function up to 20 years after diagnosis.

Figure 3: Estimated cumulative incidence of POI patients with spontaneous pregnancy up to 20 years after diagnosis

Figure 4: Survival distribution function for the time of the arrest of resumption of ovarian function in POI patients. 
Table 1: biochemical data at initial evaluation of the 117 patients with resumption of ovarian function

\begin{tabular}{|l|c|}
\hline & Mean \pm sd \\
\hline FSH (UI/l) & $54 \pm 15$ \\
\hline LH (UI/l) & $27 \pm 10$ \\
\hline Estradiol (pmol/l) & $279 \pm 363$ \\
\hline Total testosterone $(\mathrm{nmol} / \mathrm{l})$ & $0.90 \pm 0.49$ \\
\hline Androstenedione $(\mathrm{nmol} / \mathrm{l})$ & $5.0 \pm 2.3$ \\
\hline DHEA (nmol/l) & $24.3 \pm 50.3$ \\
\hline AMH $(\mu \mathrm{g} / \mathrm{l})$ & $0.3 \pm 0.8$ \\
\hline Inhibin B (ng/l) & $22 \pm 42$ \\
\hline
\end{tabular}


Table 2: Univariate analysis of the arrest of ovarian function resumption in POI patients

\begin{tabular}{|c|c|c|c|}
\hline Variables & Hazard Ratio & Confidence Interval 95\% & p value \\
\hline Familial case of POI & 0.56 & $0.27-1.14$ & 0.11 \\
\hline Auto-immune disease & 1.71 & $0.87-3.36$ & 0.12 \\
\hline Tobacco use & 0.82 & $0.45-1.48$ & 0.51 \\
\hline Primary amenorrhea & 0.62 & $0.08-4.56$ & 0.64 \\
\hline Pubertal development & 0.86 & $0.12-6.25$ & 0.88 \\
\hline Pregnancy before POI & 0.96 & $0.55-1.66$ & 0.87 \\
\hline $\begin{array}{l}\text { Ovarian resumption } \\
\text { with menstrual cycles }\end{array}$ & 0.73 & $0.31-1.71$ & 0.47 \\
\hline $\begin{array}{l}\text { Ovarian resumption } \\
\text { with } \mathrm{FSH}<15 \mathrm{UI} / \mathrm{L}\end{array}$ & 0.63 & $0.37-1.08$ & 0.09 \\
\hline $\begin{array}{l}\text { Ovarian resumption } \\
\text { with pregnancy }\end{array}$ & 1.25 & $0.64-2.42$ & 0.51 \\
\hline Age at diagnosis (years) & 1.04 & 0.99-1.09 & 0.08 \\
\hline Age of menarche (years) & 1.16 & $0.99-1.37$ & 0.07 \\
\hline Age of menopause in the mother (years) & 1.05 & $0.99-1.12$ & 0.09 \\
\hline $\begin{array}{l}\text { Duration between POI diagnosis and POI } \\
\text { resumption (years) }\end{array}$ & 0.99 & $0.89-1.11$ & 0.93 \\
\hline FSH at B1 (per $100 \mathrm{UI} / \mathrm{l})$ & 1.01 & $1.001-1.011$ & 0.026 \\
\hline LH at B1 (per $100 \mathrm{UI} / \mathrm{l})$ & 2.79 & $0.86-9.05$ & 0.08 \\
\hline Estradiol at B1 (per 367 pmol/l) & 1.00 & $0.99-1.01$ & 0.81 \\
\hline Total testosterone at B1 (per 347 nmol/l) & 1.11 & $0.00-4.46$ & 0.41 \\
\hline Androstenedione at B1 (per 349 nmol/l) & 0.002 & $0.0-3.98$ & 0.81 \\
\hline DHEA at B1 (per $347 \mathrm{nmol} / \mathrm{l})$ & 1.02 & $1.003-1.036$ & 0.024 \\
\hline $\mathrm{AMH}$ at $\mathrm{B} 1(\mu \mathrm{g} / \mathrm{l})$ & 1.47 & $1.47-2.18$ & 0.06 \\
\hline Inhibin B at B1 (per 100 ng/l) & 1.00 & $0.99-1.01$ & 0.97 \\
\hline Presence of follicles at ultrasonography & 1.045 & $0.586-1.220$ & 0.21 \\
\hline
\end{tabular}

B1: initial evaluation. 


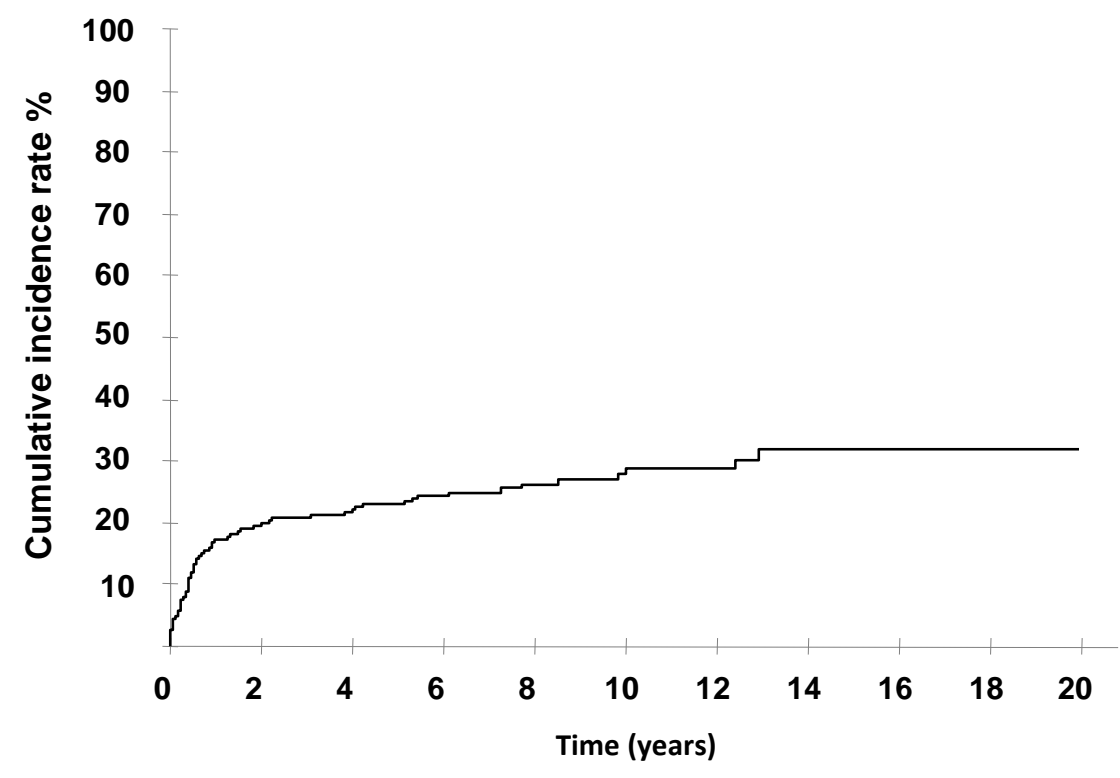

Figure 2 


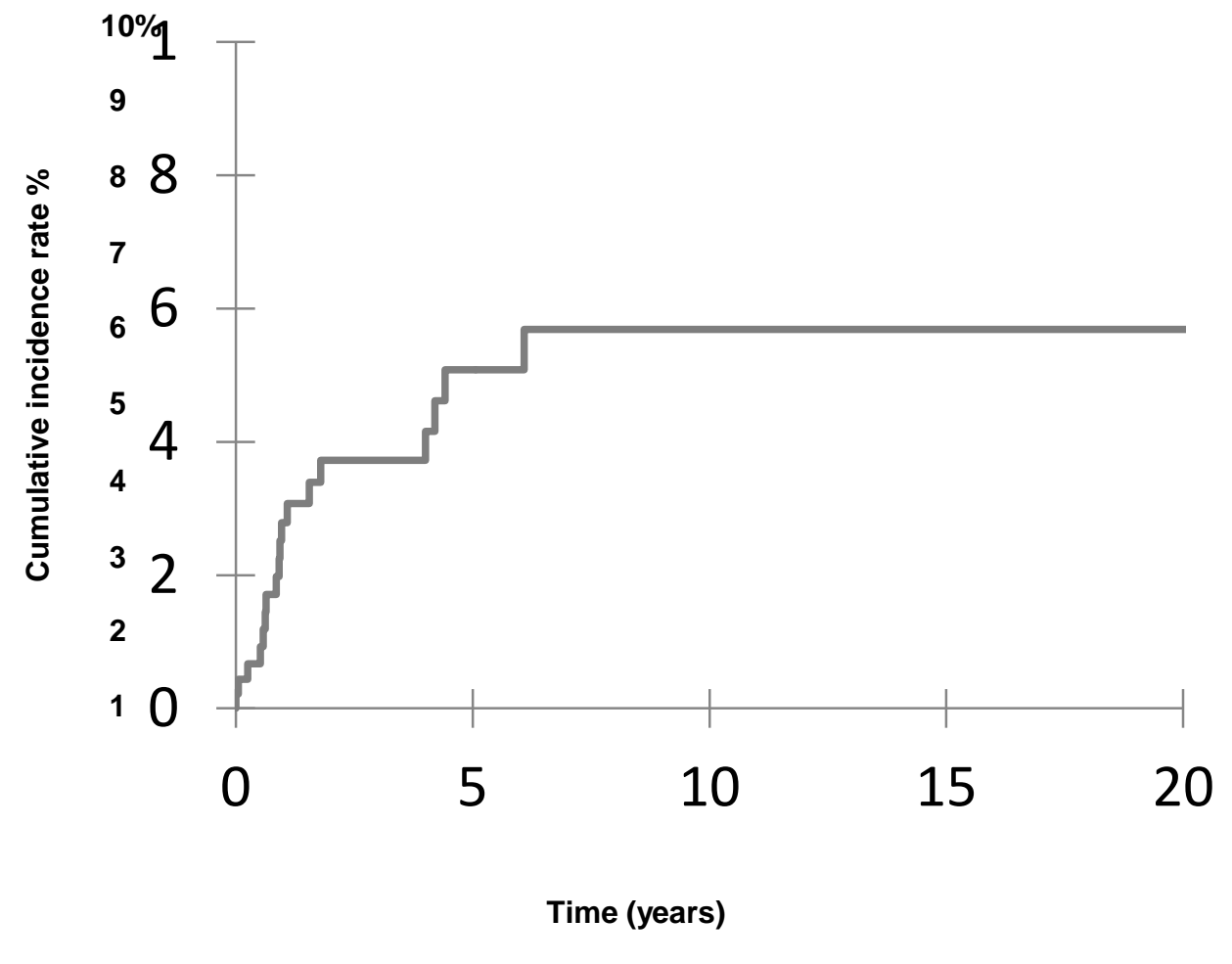

Figure 3 


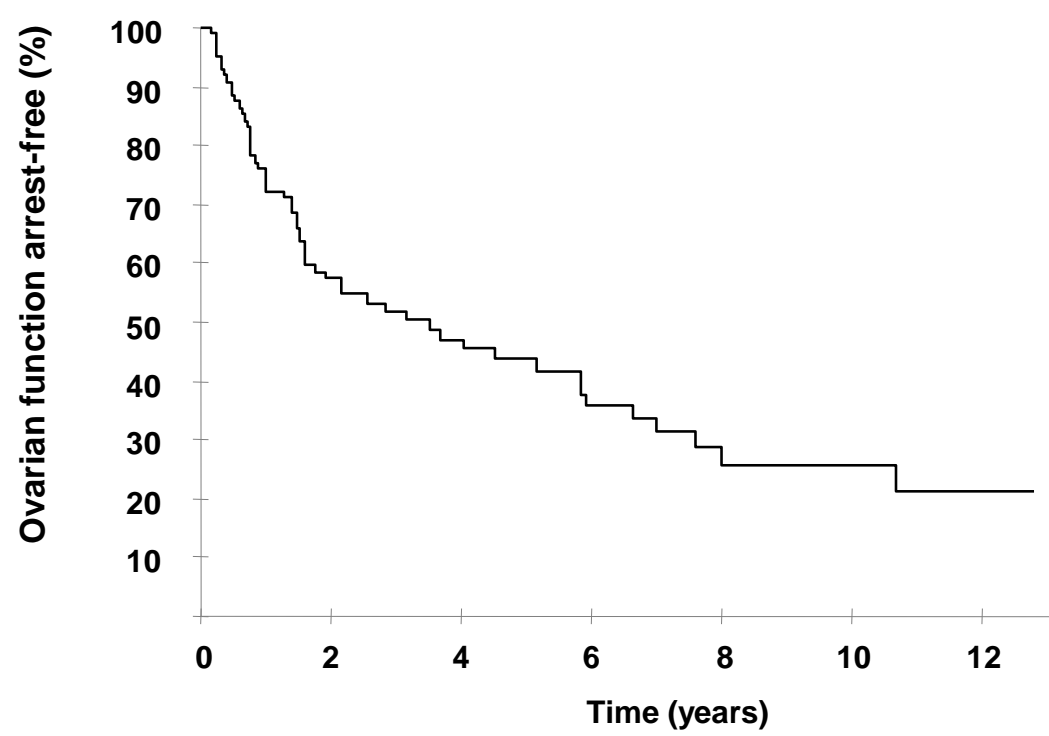

Figure 4 